\title{
416 - Risk of Mortality Associated with Antipsychotics in Alzheimer's Disease
}

Liliana P. Ferreira1, Núria Santos1, Nuno Fernandes1, Carla Ferreira2

1.Hospital Distrital de Santarém, E.P.E.

2.Centro Hospitalar Lisboa Norte - Hospital Santa Maria

Objectives: Alzheimer's disease (AD) is the most common cause of dementia and it is associated with increased mortality. The use of antipsychotics is common among the elderly, especially in those with dementia. Evidence suggests an increased risk of mortality associated with antipsychotic use. Despite the short-term benefit of antipsychotic treatment to reduce the behavioral and psychological symptoms of dementia, it increases the risk of mortality in patients with AD. Our aim is to discuss the findings from the literature about risk of mortality associated with the use of antipsychotics in AD.

Methods: We searched Internet databases indexed at MEDLINE using following MeSH terms:

"Antipsychotic Agents" AND "Alzheimer Disease" OR "Dementia" AND "Mortality" and selected articles published in the last 5 years.

Results: Antipsychotics are widely used in the pharmacological treatment of agitation and aggression in elderly patients with $A D$, but their benefit is limited. Serious adverse events associated with antipsychotics include increased risk of death. The risk of mortality is associated with both typical and atypical antipsychotics. Antipsychotic polypharmacy is associated with a higher mortality risk than monotherapy and should be avoided. The mortality risk increases after the first few days of treatment, gradually reducing but continues to increase after two years of treatment. Haloperidol is associated with a higher mortality risk and quetiapine with a lower risk than risperidone.

Conclusions: If the use of antipsychotics is considered necessary, the lowest effective dose should be chosen and the duration should be limited because the mortality risk remains high with long-term use. The risk / benefit should be considered when choosing the antipsychotic. Further studies on the efficacy and risk of adverse events with antipsychotics are needed for a better choice of treatment and adequate monitoring with risk reduction.

Keywords: Alzheimer's disease, mortality, dementia, antipsychotic agents, neuroleptics. 